\title{
ASSESSMENT OF PRE-TREATMENT OF EDIBLE COATINGS PREPARED FROM GELATINE AND POLYPHENOLS (EXTRACTED UNDER OPTIMIZED CONDITIONS FROM AMLA FRUIT POWDER) ON STABILITY OF BANANA CHIPS
}

\author{
P. Mishra* and D. KALITA \\ Department of Food Engineering \& Technology, Tezpur University, Napaam, Assam-784028. India
}

(Received: 8 May 2016; accepted: 17 September 2016)

\begin{abstract}
Chakaiya variety of amla was investigated for its phenolic contents, and extracted phenolics from amla powder were evaluated for their antioxidant activity. RSM (response surface methodology) was used to determine the optimum conditions for maximum recovery of total phenolic content (TPC) and 2,6-diphenyl picryl hydrazil free radical $\left(\mathrm{DPPH}^{*}\right)$ scavenging activity. The gelatine coating on banana slices before frying significantly reduced (approximately $60 \%$ ) the fat uptake from oil during frying in comparison to control. The reduction of particle size to the nano range was found to be effective to load the antioxidants effectively. Antioxidant extracted from amla reduced the rate of oxidation of oil during frying and so it may be a better alternative of synthetic antioxidant in food products.
\end{abstract}

Keywords: amla, antioxidants, banana, gelatine, nanoparticle, edible coating

Frying involves different chemical changes, such as gelatinization of starch, denaturation of protein, browning, etc. Deep fat frying causes absorption of fat, which could be up to $50 \%$ of the total weight of fried foods (Pinthus et al., 1993). Various parameters, like time and temperature, porosity of the food material, composition (protein, fat, carbohydrate), and quality of oil, significantly affect the uptake of oil during frying. Thus, efforts were taken by scientist to reduce the absorption of fat during frying of chickpeas, potato strips, chicken nuggets, etc. Edible film and coating might protect food from oil absorption during frying, which occurs during the first frying period as water evaporation occurs (SоTHORNvit, 2011). Gelatine is widely used as a multifunctional additive, which improves stability and capable of modifying texture. Oxidative stability of the oil during frying is another important issue that cannot be ignored. Synthetic antioxidants used to prevent oxidation of food sample have also several health issues that cannot be overlooked.

Amla (Emblica officinalis), belonging to the family of Euphorbiaceae, have long been used as folk medicines in India and several other countries in curing numerous diseases (CALIXTo et al., 1998). Phenolics are one of the major constituents present in amla (KusIRISIN et al., 2009); being secondary metabolites, phenolics include a huge range of compounds like flavonols, ellagic acid, anthocyanins, and their derivatives (Luo et al., 2011), and these phenolics vary depending on species and location. So in the present investigation, extraction of phenolics from amla were optimized by using response surface methodology (RSM).

Nanoparticles have several advantages over microparticles because of their size, which leads to proportionally larger surface area and consequently more surface atoms (DE AzEREDO, 2009). Application of nano and micro-range gelatine and ethanol extracted amla powder has

* To whom correspondence should be addressed.

Phone: +912712-275705; e-mails: mpoonam1@rediffmail.com; poonam@tezu.ernet.in 
never been evaluated as an edible coating material on banana chips. In present work, the extraction conditions for the maximum recovery of phenolics were optimized by using response surface methodology (RSM). The freeze dried, ethanol extracted (under optimal conditions) amla powder was loaded on gelatine nanoparticles to form an edible coating having antioxidant properties. The effect of coating on the stability of oil during frying of banana chips was investigated.

\section{Materials and methods}

\subsection{Raw material}

Amla of Chakaiya variety was procured from local market of Allahabad, India. Cleaned fruit were grated and dried at $40{ }^{\circ} \mathrm{C}$. Dried amla shreds were ground in laboratory mill to pass through a $0.5 \mathrm{~mm}$ sieve. The collected powder was packed in air tight container and stored at refrigerated temperature for further analysis.

\subsection{Optimization of extraction of total phenolics from amla fruit powder by RSM}

RSM was used to evaluate the effect of variables on total recovery of phenolic content and $\mathrm{DPPH}^{*}$ radical scavenging activity. Preliminary study was conducted to decide the level of $\mathrm{pH}$, temperature, and ethanol concentration. A central composite rotatable (CCD) design with three numeric factors on five levels was used for designing the experimental conditions. Temperature $\left(\mathrm{x}_{1}\right)$, ethanol ratio in ethanol water mixture $\left(\mathrm{x}_{2}\right)$, and $\mathrm{pH}\left(\mathrm{x}_{3}\right)$ were selected as independent variables. The level of variation is made in ethanol ratio from $10-90 \%$; temperature from 15 to $55^{\circ} \mathrm{C}$, and $\mathrm{pH}$ from 4 to 8 in all 20 experiments included in the design for the extraction. Amla powder weighing $1 \mathrm{~g}$ was transferred into a 50 -ml conical flask and $10 \mathrm{ml}$ (constant volume) of an ethanol water mixture was added. The sample mixture was stirred in an incubator shaker at 160 r.p.m. for $4 \mathrm{~h}$ at selected temperature. The sample mixture was centrifuged at 8000 r.p.m. in refrigerated centrifuge for $10 \mathrm{~min}$ at $4{ }^{\circ} \mathrm{C}$ temperature. The supernatant was carefully collected and stored at $-20^{\circ} \mathrm{C}$ temperature for further analysis of TPC and DPPH* radical scavenging activity. Extraction parameters were normalized as coded variables so they affect the response more evenly and so the units of parameters irrelevant (BAS \& BOYACI, 2007). Variables were coded as per the formula given below:

$$
X=\frac{\left(x_{i}-x_{0}\right)}{\Delta x}
$$

where $X$ is the coded value, $x_{\mathrm{i}}$ is the actual value, $\mathrm{x}_{\mathrm{o}}$ is the actual value in the centre of domain, and $\Delta x$ is the increment of $x_{\mathrm{i}}$ corresponding to variation in one unit of $x$. Response variables were fitted with polynomial model equation (given below), which is generally able to describe the relationship between the responses and the independent variables.

$$
Y=\beta_{0}+\sum_{i=1}^{2} \beta_{i} X_{i}+\sum_{i=1}^{2} \beta_{i i} X_{i}^{2}+\sum_{i<j=1}^{2} \beta_{i j} X_{i} X_{j}
$$

where $Y$ represents the response variables, $X_{\mathrm{i}}$ and $X_{\mathrm{j}}$ represent independent variables, and $\beta_{0}$, $\beta_{\mathrm{i}}, \beta_{\mathrm{ii}}$, and $\beta_{\mathrm{ij}}$ represent the regression coefficient for intercept, linear, quadratic, and interaction terms, respectively. Analysis of variance (ANOVA) was used in order to evaluate model adequacy, regression coefficients, and statistical significance. Statistical analysis was 
performed using RSM software Design matrix version 7.0.0 trial (Stat-Ease, Minneapolis, $\mathrm{MN}$, USA). The results reported were at $\mathrm{P}=0.05$. The model adequacy was tested by coefficient of determination $\left(\mathrm{R}^{2}\right)$, model $\mathrm{P}$ value, and lack of fit testing.

\subsection{Analysis of bioactive properties of amla}

DPPH* scavenging activity and total phenolic content (TPC) by Folin-Ciocalteu method were performed by the method of Liu and co-workers (2012).

\subsection{Preparation of gelatine nanoparticles}

Gelatine nanoparticles were prepared by using the desolvation technique as per the method given by Jun and co-workers (2011). Gelatine ( $1 \mathrm{~g})$ was added to double distilled water, and subsequently the $\mathrm{pH}$ of the solutions were adjusted (to 7 or 9) using $1 \mathrm{M} \mathrm{NaOH}$ solution. The solutions were stirred continuously for $8 \mathrm{~h}$ at $500 \mathrm{r}$.p.m. in a magnetic stirrer for complete hydration. Then acetone was added in a dropwise manner to the gelatine solution at a rate of $1 \mathrm{ml} \mathrm{min}{ }^{-1}$ till the solution attained turbidity. Further $0.01 \mathrm{ml}$ of $4 \%$ calcium chloride in ethanol solution was added to the gelatine solution to induce intra-particle cross linking. After continuous stirring for $3 \mathrm{~h}$, the gelatine solution was purified by 2 cycles of centrifugation at 6000 r.p.m. for $30 \mathrm{~min}$ (Sigma 3-18 K Germany). After centrifugation, the pellets were redispersed to the original volume of absolute ethanol using an ultrasonicator and then stored at $4{ }^{\circ} \mathrm{C}$ for further analysis.

\subsection{Preparation of nanoparticle coating}

Gelatine nanoparticles containing ethanol solution was dried at $40{ }^{\circ} \mathrm{C}$ in a tray drier. One hundred $\mathrm{ml}$ of solution gave approximately $0.77 \mathrm{~g}$ of gelatine nanoparticle. For preparation of coating with gelatine (without any treatment), $0.13 \mathrm{~g}$ of gelatine was dissolved in $100 \mathrm{ml}$ of water and the mixture was stirred under magnetic stirrer at 500 r.p.m. till the gelatine was completely dispersed. For loading of antioxidant, two different levels of freeze dried ethanol extracted amla powder $(0.1$ and $0.3 \%$ on antioxidant powder weight/volume of gelatine solution) were dissolved in gelatine solutions and the mixture of the solution was stirred under magnetic stirrer at 500 r.p.m. for 30 min. Butylated hydroxyl toluene (BHT) was taken as a positive control to compare the effectiveness of natural antioxidants extracted from amla powder. The levels of antioxidants were decided on the basis of safe level of synthetic antioxidants used in food products.

\subsection{Preparation of banana chips}

Bananas were hand peeled, washed, and cut into strips of $0.4 \mathrm{~cm}$ thickness with a lab scale slicer. The banana used for the present study contained $18.23 \%$ carbohydrate and $0.23 \%$ fat. The banana slices were washed with water and were blanched for $3 \mathrm{~s}$ at $85^{\circ} \mathrm{C}$ in $0.5 \%$ citric acid solution. After blanching, the strips of banana were dipped in the coating solution at the ratio of 1:10 of banana slices:coating solution ( $\mathrm{w} / \mathrm{v}$ basis) for $2 \mathrm{~min}$ at room temperature. The slices were drained and dried in hot air oven at $105^{\circ} \mathrm{C}$ for $10 \mathrm{~min}$ to remove surface moisture. The frying of banana chips was carried out in an oil fryer at $140{ }^{\circ} \mathrm{C}$ for $3 \mathrm{~min}$ in edible coconut oil with constant product weight:oil volume ratio of 1:30 (SingTHONG \& THONGKAEW, 2009). The fried samples were allowed to cool and were packed in high density film for further analysis. For control, banana chips were dipped in water instead of coating solution 
for two minutes. Enzymatic browning is a commonly occurring phenomenon for banana, so to avoid the enzymatic browning of the control sample, banana slices were dipped into the water without any coating solution.

\subsection{Moisture content (MC), fat content, peroxide value (POV), and thiobarbituric acid (TBA) value of extracted fat}

The fat of fried banana chips was extracted using Soxtec apparatus (Pelican, India) by using petroleum ether as solvent at $35 \pm 1^{\circ} \mathrm{C}$ as per the method of AOAC (2010) with three replicates. Extracted fats were analysed for moisture content (oven drying method), fat content, POV, and TBA as per the method of RANGANNA (1986).

\subsection{Colour characteristics of banana chips}

The colour characteristics of the banana chips were determined by using Hunter color lab UltraScan VIS (USA) colour measurement spectrophotometer. Obtained results were expressed in terms of $L, a$, and $b$, where $L$ denotes lightness vs. darkness, $a$ redness vs. greenness, and $b$ yellowness vs. blueness.

\subsection{Statistical analysis}

All experiments were performed in duplicate, the mean value and standard deviation were calculated using Excel version of 2003. Statistical analysis was performed using RSM software Design matrix version 7.0.0 trial (Stat-Ease, Minneapolis, MN, USA). The results were reported at $\mathrm{P}=0.05$.

\section{Results and discussion}

\subsection{Optimization of extraction of phenolics}

Figure 1A.a shows the effect of ethanol concentration and extraction temperature on TPC of the extract in terms of gallic acid equivalent. Increasing the ethanol concentration increased the recovery of TPC significantly. Especially, when the ethanol concentration was increased from 10 to $72 \%$, the TPC per $\mathrm{ml}$ of the extract increased. Maximum phenolics were recovered at approximately $71.30 \%$ of ethanol concentration. The interactive effect of temperature and ethanol concentration was not found statistically significant for recovery of TPC. Similar trend was observed for $\mathrm{DPPH}^{*}$ scavenging activity of the extract, with an increase in the concentration of the ethanol from 10 to $74 \%$, a significant increase in the $\mathrm{DPPH}^{*}$ scavenging activity of the extract was found, and beyond that, a slight decrease in the DPPH* scavenging activity was observed (Fig. 1A.b). The present findings are in accordance with the conclusions of BuCIC-KoJIC and co-workers (2009), that the aqueous solution of ethanol is more effective than absolute ethanol in extracting polyphenols. Similar results were also reported by SHI and co-workers (2003), that the absolute ethanol and pure water are comparatively less effective for the extraction of phenolic content than mixture of ethanol and water. The DPPH* scavenging activity of the extract increased with the increase of extraction temperature up to $40{ }^{\circ} \mathrm{C}$, but beyond that a reverse trend was observed (Fig. 1A.b). MisHRA and co-workers (2015a) also found that the exposure to higher temperature may adversely affect the structure of phenolic content, thus reducing the $\mathrm{DPPH}^{*}$ scavenging activity. The interactive effect of temperature and ethanol concentration was found statistically significant (Fig. 1B.a). 
Increasing the $\mathrm{pH}$ value up to 6 slightly increased the DPPH* scavenging activity, whereas further increase in the $\mathrm{pH}$ reduced the efficiency of the extracts to scavenge the DPPH* (Figs 1B.b, 1C.a). This observation was consistent with our previous findings (Mishra et. al., 2015b). Figure 1C.b depicts the 3D plot, showing the effect of ethanol concentration and $\mathrm{pH}$ on \% $\mathrm{DPPH}^{*}$ scavenging activity of the extract. Increasing the concentration of ethanol at lower $\mathrm{pH}$ increased the \% $\mathrm{DPPH}^{*}$ scavenging activity of the extract significantly, while increasing the $\mathrm{pH}$ showed an adverse effect on DPPH* scavenging activity of the extract.

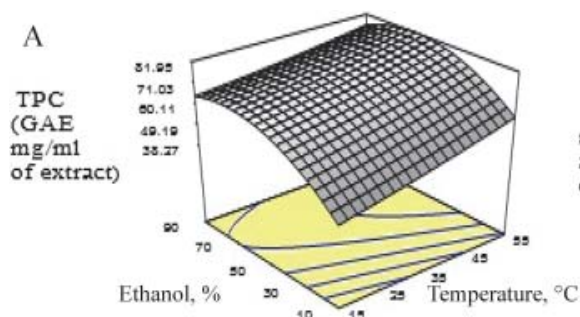

(a)

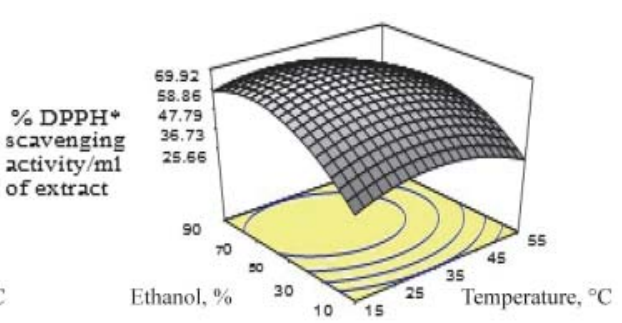

(b)

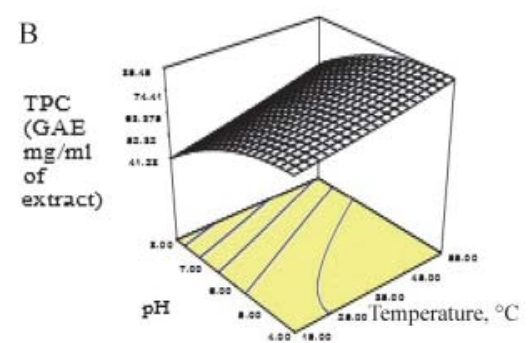

(a)

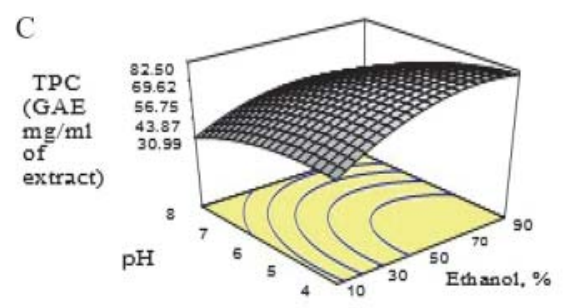

(a)

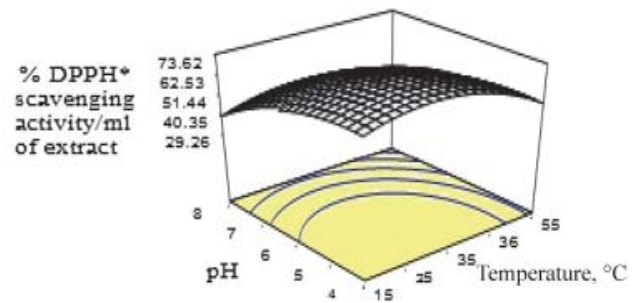

(b)

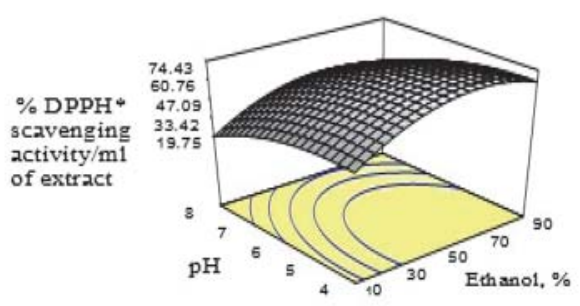

(b)

Fig. 1 A: Response surface and contour plots for (a) total phenolic content, and (b) DPPH* scavenging activity (at constant $\mathrm{pH}$ 6.0)

B: Response surface and contour plots for (a) total phenolic content, and (b) DPPH* scavenging activity (at constant ethanol concentration of $50.0 \%$ ).

C: Response surface and contour plots for (a) total phenolic content, and (b) DPPH* scavenging activity (at constant temperature $35^{\circ} \mathrm{C}$ ) 
All obtained results were fitted with a second-order polynomial model, and multiple regression coefficients were generated for all responses, using method of least square approach (MLS). Regression coefficients and their statistical significance for each TPC and $\mathrm{DPPH}^{*}$ radical scavenging activity are summarized in Table 1A. Model adequacy was estimated by calculating the $\mathrm{F}$ values for model and for lack of fit test and by $\mathrm{R}^{2}$. Particularly high $\mathrm{R}^{2}$ for both TPC and $\mathrm{DPPH}^{*}$ confirmed that the applied polynomial models were in accordance with the experimental results. Considering all responses obtained simultaneously, RSM was used to compromise optimum conditions. Optimized conditions of variables for maximum recovery of TPC and DPPH* radical scavenging activity of extract were obtained by numerical optimization techniques. Ethanol $78.0 \%$, temperature $30.5{ }^{\circ} \mathrm{C}$, and $4.5 \mathrm{pH}$ were observed as the optimum conditions with 0.98 desirability for both TPC and DPPH* scavenging activity (Table 1B).

Table 1A. Regression coefficients ( $\beta$ ), coefficient of determination $\mathrm{R}^{2}$, and $\mathrm{F}$-test value of predicted second order polynomial models for TPC and DPPH* scavenging activity

\begin{tabular}{lcc}
\hline Regression coefficients & TPC & DPPH* radical scavenging activity \\
\hline Intercepts & 69.25 & 64.87 \\
$\mathrm{X}_{\mathrm{o}}$ & & \\
Linear & 8.13 & -5.82 \\
$\beta_{1}$ & -13.84 & -13.23 \\
$\beta_{2}$ & +10.82 & +10.15 \\
$\beta_{3}$ & & \\
Quadratic & +1.64 & -11.09 \\
$\beta_{11}$ & -6.64 & -7.55 \\
$\beta_{12}$ & -12.61 & -16.16 \\
$\beta_{33}$ & & \\
Cross product & +1.77 & +1.27 \\
$\beta_{11}$ & -2.24 & -3.19 \\
$\beta_{12}$ & -4.45 & -1.14 \\
$\beta_{13}$ & 0.9668 & 0.9561 \\
$\mathrm{R}^{2}$ & 26.25 & 24.21 \\
F value (model) & 4.74 & 3.61 \\
F value (lack of fit) & & \\
\hline
\end{tabular}

Table 1B. Extraction of total phenols and \% DPPH* scavenging activity under optimum conditions

\begin{tabular}{lcc}
\hline Particular & Predicted & Optimum \\
\hline \% DPPH* scavenging activity $/ \mathrm{ml}$ of extract & 67.50 & 68.50 \\
Total phenolic content as $\mathrm{mg} \mathrm{GAE} / \mathrm{ml}$ of extract & 71.60 & 73.60 \\
\hline
\end{tabular}




\subsection{Effect of coating on quality attributes of banana chips}

2.2.1. Effect of coating on oil absorption of fried banana chips. Coating of banana slices with gelatine and amla extracted powder produced a significant reduction in oil absorption in comparison to control, as reflected in Figure 2B.a. Control sample absorbed $37.97 \%$ oil during frying, however, treated samples absorbed oil in the range of 8.96 to $16.83 \%$ oil. Sample treated with gelatine of reduced particle size was comparatively more effective to reduce the fat uptake in fried banana chip than gelatine (without any modification in size), however, addition of amla extracted powder showed no significant effect on oil uptake of banana chips. Since fat uptake was significantly less in the sample treated with gelatine of $100 \mathrm{~nm}$ particle size, only the gelatine of $100 \mathrm{~nm}$ particle size was used in further studies.

Oil absorption occurs during the cooling phase, and is known as the vacuum effect (SHYu \& HwANG, 2011). After complete frying, the product starts to cool, water vapour condenses, which consequently decreases the internal pressure and adhered oil on food surfaces is sucked into the void spaces of the food. So from the above theory given by the scientists, it was hypothesized that the application of coating of gelatine interferes with the surface phenomena of absorption of oil, and subsequently, significant reduction in absorption of oil was observed. Reducing the size of gelatine, the surface area was significantly increased, and so the coating was uniformly distributed on the banana slices, which reduced the oil uptake during frying. Incorporation of amla derived antioxidant showed no obvious effect on fat uptake.

2.2.2. Effect of coatings on moisture content $(M C)$. Coating of banana chips showed significant effect on retention of MC (Fig. 2B.b). All pre-treated slices had comparatively more retention of moisture (4.8-5.1\%) than the control (3.9\%). A reciprocal relationship between moisture loss and oil absorption was observed by AKDENIZ and co-workers (2006). Gum coating showed effective moisture retention due to the strong interaction of hydrogen bonds between water molecules, which causes lower oil absorption (AKDENIZ et al., 2006). In this study, it was observed that lower oil uptake correlated with moisture loss, which supports the findings of Pinthus and co-workers (1993). Added antioxidants could not alter the functionality of gelatine in terms of water holding properties, and so they had no further effect on the moisture content of fried banana chips. SOTHORNVIT (2011) also reported effective moisture retention and lower oil absorption in banana chips when pre-treated with hydrocolloids before frying.

2.2.3. Effect of coating on oxidative stability of fat extracted from fried banana chips. The effects of coating on POV of fat extracted from fried banana chips are depicted in Figure 2B.c. Significant reduction in POV was observed in the fat extracted from pre-treated banana chips when compared with control. Reduction of size of gelatine before applying as coating material was significantly effective in protecting the oil from oxidation. Fat extracted from banana slices treated with gelatine $(100 \mathrm{~nm})$ and antioxidants had comparatively less POV than any other sample tested. BHT and extracted antioxidant from amla were found to be effective in reducing the POV in a dose dependent manner. Addition of antioxidant either derived from natural or synthetic origin had noticeable effect on the rate of oxidation during frying. Antioxidants loaded on gelatine of reduced particle size effectively control the increase of POV during frying. This may be attributed to the small size of the nanoparticles, which leads to an increase in the surface area making loading and distribution of antioxidants more efficient (DE AZEREDO, 2009). The present finding supports the findings of VINSON and co-workers (2002), that plant extracts obtained from some fruit and vegetable have effective antioxidant properties. 
A

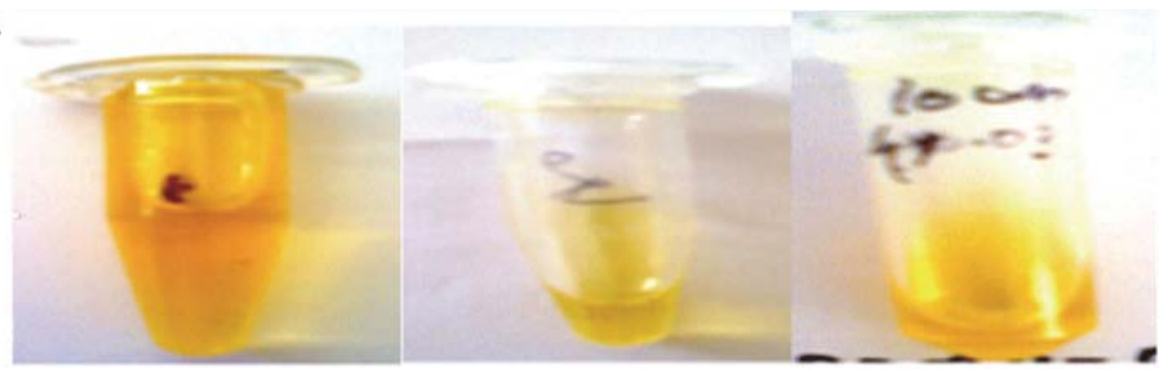

(a)

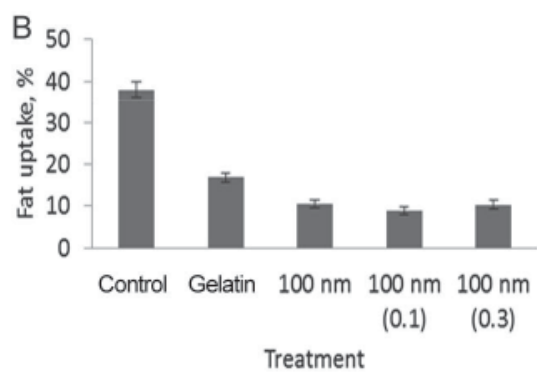

(a)

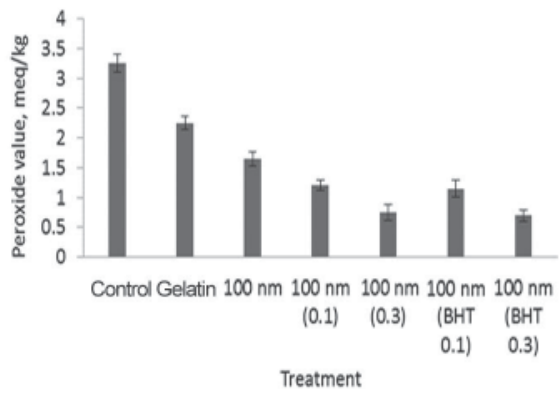

(c) (b)

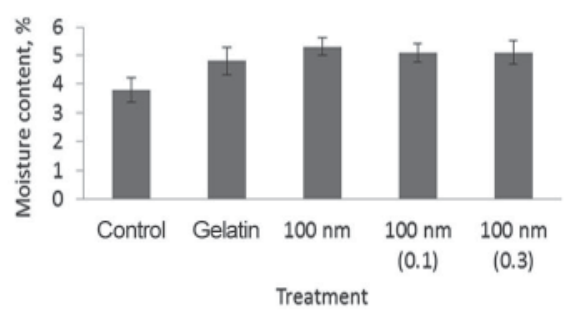

(b)

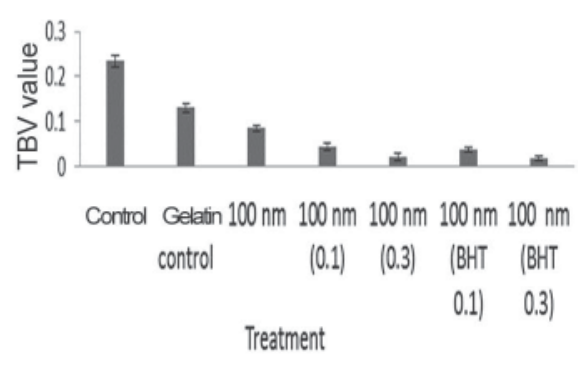

(d)

Fig. 2. A: Images of fat extracted from the same amount of fried banana chips (a) control (b) pre-treated banana with gelatine $(100 \mathrm{~nm})+0.1 \%$ extracted amla powder, (c) pre-treated banana with gelatine $(100 \mathrm{~nm})+0.3 \%$ extracted amla powder

B: Effect of coating on (a) fat uptake of banana chips and (b) moisture content of fried banana chips (c) on POV of fat extracted from fried banana chips and (d) on TBA value of extracted fat

The effect of coating on TBA value of extracted fat is depicted in Fig 2B.d. Similar trend could be observed as that in the case of POV, i.e. nanoparticles containing coating were effective in lowering the TBA value of banana chips. The coating loaded with natural antioxidant of $0.3 \%$ was comparatively more effective than BHT at $0.1 \%$ concentration. 
2.2.4. Colour characteristics of fried banana chips. In general, the treatment significantly affected $a$ value of the fried banana chips (Fig. 3b). $L$ values of all samples were very close to each other with the exception of the sample treated with gelatine only, which suggests that treatment of the sample with gelatine nanoparticle has no adverse effect on the lightness of the sample (Fig. 3a). The redness of the sample treated with extracted amla powder containing coating was comparatively high compared with the other sample tested. The probable reason of the increased value of $a$ might be the presence of tannin in extracted amla powder, which may convert into iron tannates during frying increasing the redness of the samples (Fig. 3b). Loss of redness (lowest $\mathrm{a} / \mathrm{b}$ value) was found in the sample treated by gelatin only (Fig. 3d). Redness of the control sample may be related to the low moisture content of the sample, which may promote Maillard and caramelization reactions increasing the redness of the sample. Application of gelatine coatings (without any modification) increased the yellowness in fried banana chips (Fig. 3c) and increased the $L$ of the sample (Sothornvit, 2011).

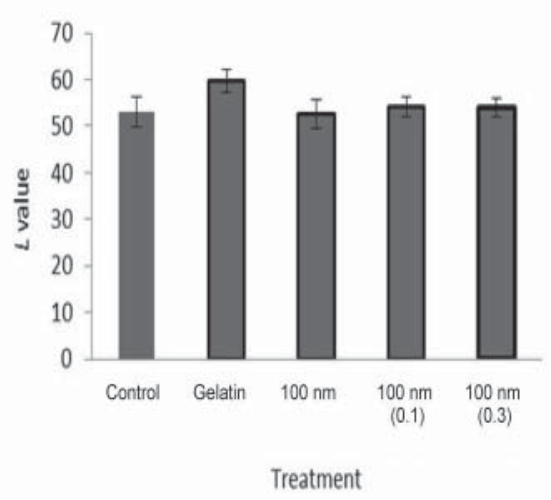

(a)

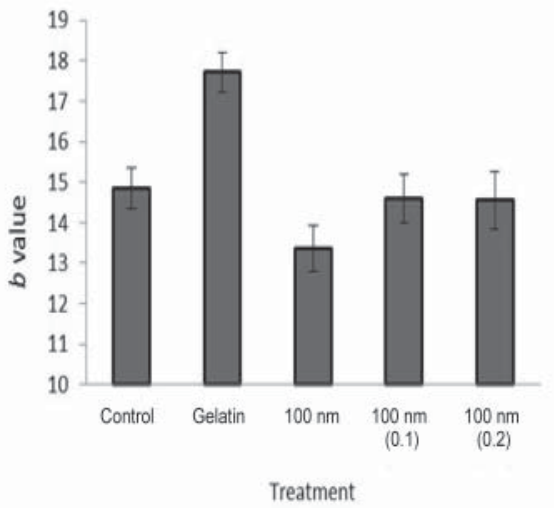

(c)

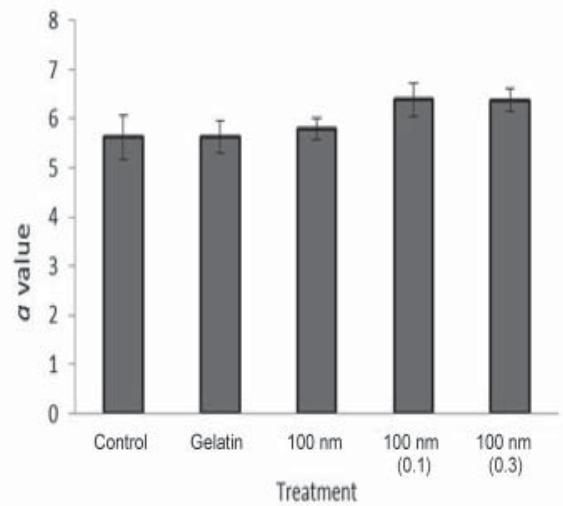

(b)

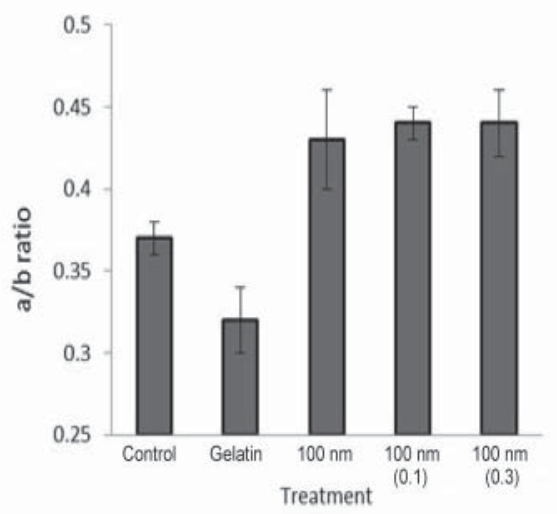

(d)

Fig. 3. Effect of coating on colour attributes of banana chips during frying (a) $L$ value, (b) $a$ value, (c) $b$ value, and $(d) a / b$ ratio 


\section{Conclusions}

RSM (response surface methodology) was used to determine the optimum conditions for maximum recovery of TPC and DPPH* scavenging activity. The gelatine coating on banana slices before frying significantly reduced the fat uptake during frying of banana. The reduction of size of particles to nano made antioxidant loading effective. Antioxidant extracted from amla reduced the rate of oxidation of edible oil during frying and so it may be a better alternative of synthetic antioxidants in food products.

\section{References}

AOAC (2010): Approved methods of analysis, 18th ed. Association of Official Analytical Chemists, Washington, USA. Method No. 2003.05.

Akdeniz, N., Sahim, S. \& Sumnu, G. (2006): Functionality of batters containing different gums for deep fat frying of carrot slices. J. Food Eng., 75, 522-526.

BAs, D. \& Boyaci, I.H. (2007): Modelling and optimization I: Usability of response surface methodology. J. Food Eng., 78, 836-845.

Bucic-Kojic, A., Planinic, M., Tomas, S., Jackobek, L. \& Seruga, M. (2009): Influence of solvent and temperature on extraction of phenolic compounds from grape seed, antioxidant activity and colour of extract. Int. J. Food Sci. Tech., 44, 2394-2401.

Calixto, J.B., Santos, A.R., Cechinel, F.V. \& Yunes, R. (1998): A review of the plants of the genus Phyllanthus: their chemistry, pharmacology, and therapeutic potential. Med. Res. Rev., 18, 225-258.

DE Azeredo, M.C.H. (2009): Nanocomposite for food packaging applications. Food Res. Int., 42, 1240-1253.

Jun, J.Y., Nguyen, H.H., Paik, R.Y.S., Chu, S.H., Kang, C.B. \& Ko, S. (2011): Preparation of size-controlled bovine serum albumin (BSA) nanoparticles by a modified desolvation method. Food Chem., 127, 1892-1898.

Kusirisin, W., Srichairatanakool, S., Lerttrakarnnon, P., Lailerd, N., Suttajit, M. \& Jaikang, C. (2009): Antioxidative activity, polyphenolic content and antiglycation effect of some Thai medicinal plants traditionally used in diabetic patients. Med. Chem., 5, 139-147.

Liu, X., Zhao, M., Wu, K., Chai, K., Yu, H., TAo, Z. \& WANG, J. (2012): Immunomodulatory and anticancer activities of phenolics from emblica fruit (Phyllanthus emblica L.). Food Chem., 131, 685-690.

Luo, W., Zhao, M., Yang, B., Ren, J., Shen, G. \& Rao, G. (2011): Antioxidant and antiproliferative capacities of phenolics purified from Phyllanthus emblica L. fruit. Food Chem., 126, 277-282.

Mishra, P., Dutta, N. \& Mahanta, C. (2015a): Partial extraction and identification of phenolics in amla (Emblica officinalis) seed coat powder. J. Food Sci. Tech., 52, 6990-7001.

Mishra, P., Rai, G.K. \& Mahanta, C. (2015b): Process standardization for development of spray-dried lemon juice powder and optimization of amla-lemon based RTS (ready-to-serve) drink using response surface methodology. J. Food Process. Pres., 39, 1216-1218.

Pinthus, E.J., Weinberg, P. \& Saguy, I.S. (1993): For oil uptake during deep fat frying. J. Food Sci., 58, $204-205$.

Ranganna, S. (1986): Handbook of analysis and quality control for fruit and vegetable products. Tata McGraw-Hill Education. New Delhi, pp. 3-4, 10-12 and 237-238.

Shi, J., Yu, Pohorly, J., Young, C.J., Bryan, M. \& Wu, Y. (2003): Optimization of the extraction of polyphenols from grape seed meal by aqueous ethanol solution. J. Food Agic. Environ. 1(2), 42-47.

Shyu, S.L. \& Hwang, L.S. (2011): Effects of processing conditions on the quality of vacuum fried apple chips. Food Res. Int., 34, 133-142.

Singthong, J. \& ThongKaew, C. (2009): Using hydrocolloids to decrease oil absorption in banana chips. LWT-Food Sci. Technol., 42, 1199-1203.

Sothornvit, R. (2011): Edible coating and post-frying centrifuge step effect on quality of vacuum-fried banana chips. J. Food Eng., 107, 319-325.

Vinson, J.A., Su, X., Zubik, L. \& Bose, P. (2002): Phenol antioxidant quantity and quality in foods: fruits. J. Agr. Food Chem., 49, 5315-5321. 